\title{
Characteristic Classes and Zeroth Order Pseudodifferential Operators
}

\author{
Andrés Larrain-Hubach, Steven Rosenberg, Simon Scott, \\ and Fabián Torres-Ardila
}

\begin{abstract}
We provide evidence for the conjecture that the Wodzicki-Chern classes vanish for all bundles with invertible zeroth order pseudodifferential operators $\Psi \mathrm{DO}_{0}^{*}$ as structure group. The leading order Chern classes are nonzero in general, and they detect elements of the de Rham cohomology groups $H^{*}\left(B \Psi \mathrm{DO}_{0}^{*}, \mathbb{C}\right)$.
\end{abstract}

June 7, 2018

To Misha Shubin

\section{Introduction}

Infinite dimensional manifolds $\mathcal{M}$ such as the loop space $\operatorname{Maps}\left(S^{1}, M\right)$ of a manifold or more generally the space $\operatorname{Maps}(N, M)$ of maps between manifolds have interesting geometry. The structure group of these infinite dimensional manifolds (i.e. of their tangent bundles $\mathcal{E}$ ) is a gauge group of a finite rank bundle $E \rightarrow N$ over the source space. When the manifolds have Riemannian metrics, the mapping spaces have Levi-Civita connections with connection and curvature forms taking values in $\Psi \mathrm{DO}_{\leq 0}=\Psi \mathrm{DO}_{\leq 0}(E)$, the algebra of nonpositive integer order classical pseudodifferential operators ( $\Psi$ DOs) acting on sections of $E$. Thus for geometric purposes, the structure group should be enlarged to $\Psi \mathrm{DO}_{0}^{*}$, the group of invertible zeroth order classical $\Psi D O s$, since at least formally $\operatorname{Lie}\left(\Psi \mathrm{DO}_{0}^{*}\right)=\Psi \mathrm{DO}_{\leq 0}$.

As discussed in 11], 12, 15, the generalizations of Chern-Weil theory to $\Psi \mathrm{DO}_{0}^{*}$-bundles are classified by the set of traces on $\Psi \mathrm{DO}_{\leq 0}$, i.e. by the Hochschild cohomology group $H H^{0}\left(\Psi \mathrm{DO}_{\leq 0}, \mathbb{C}\right)$. Indeed, given such a trace $T: \Psi \mathrm{DO}_{\leq 0} \rightarrow \mathbb{C}$, one defines $T$-Chern classes of a connection with curvature $\Omega \in \Lambda^{2}\left(\mathcal{M}, \Psi D \mathrm{O}_{\leq 0}\right)$ by the de Rham class $c_{k}^{T}(\mathcal{E})=\left[T\left(\Omega^{k}\right)\right] \in H^{2 k}(\mathcal{M}, \mathbb{C})$.

These traces roughly break into two classes: the Wodzicki residue and the integral of the zeroth/leading order symbol over the unit cosphere bundle. In $\S 2$, we prove that the Wodzicki-Chern classes vanish if the structure group reduces from $\Psi \mathrm{DO}_{0}^{*}$ to the subgroup with leading order symbol given by the identity. We conjecture that the Wodzicki-Chern classes always vanish, and sketch a possible superconnection proof. These vanishing results, which were previously known only

2010 Mathematics Subject Classification. Primary: 58J40.

(C)0000 (copyright holder) 
for loop spaces, reinforce the importance of the nontrivial Wodzicki-Chern-Simons classes produced in $\mathbf{1 2}$.

In the beginning of $\S 3$, we discuss the analytic and topological issues involved with universal bundle calculations of Chern classes associated to the leading order symbol trace. The main issue is that the classifying space $B \Psi \mathrm{DO}_{0}^{*}$ may not be a manifold, so we want to extend the leading order symbol trace to an algebra whose corresponding classifying space is clearly a manifold. $\S 3$ is devoted to the analytic issue of extending the leading order symbol trace to a Lie algebra containing $\Psi \mathrm{DO}_{\leq 0}$. It is easier to work with the quotient of $\Psi \mathrm{DO}_{\leq 0}$ by smoothing operators, and in Proposition 3.6 we find an extension (really a factorization) of the leading order symbol trace to a relatively large subalgebra $\mathcal{Q}$ inside a quotient of the set of all bounded operators on a Hilbert space. These results may not be optimal, so this section should be considered work in progress.

Unfortunately, the classifying space $B Q$ associated to $\mathcal{Q}$ may not be a manifold, so we are unable to construct universal geometric characteristic classes. In $\S 4$, we take a smaller extension of the leading order symbol trace with corresponding classifying space a manifold. We then show that the leading order Chern classes of gauge bundles are nontrivial in general. This implies that there is a topological theory of characteristic classes of $\Psi \mathrm{DO}_{0}^{*}$-bundles involving the cohomology of $B \Psi \mathrm{DO}_{0}^{*}$. As a first step toward understanding this cohomology, we use the nonvanishing of leading order Chern classes on mapping spaces to show in Theorem 4.10 that for $E^{\ell} \rightarrow N, H^{*}\left(B \Psi \mathrm{DO}_{0}^{*}, \mathbb{C}\right)$ surjects onto the polynomial algebra $H^{*}(B U(\ell), \mathbb{C})=\mathbb{C}\left[c_{1}(E U(\ell)), \ldots, c_{\ell}(E U(\ell))\right]$. This complements Rochon's work [16 on the homotopy groups of a certain stablilization of $\Psi \mathrm{DO}_{0}^{*}$. The proof shows that $H^{*}(B \mathcal{G}, \mathbb{C})$ also surjects onto $H^{*}(B U(\ell), \mathbb{C})$, where $\mathcal{G}$ is the gauge group of $E$. For comparison, $H^{*}\left(B \mathcal{G}_{0}, \mathbb{C}\right)$, where $\mathcal{G}_{0}$ is the group of based gauge transformations, has been completely determined by different methods, and $H^{*}(B \mathcal{G}, \mathbb{C})$ is known if the center of the underlying finite dimensional Lie group is finite [5. p. 181].

As much as possible, we sidestep the trickier analytic and topological aspects of $B \Psi \mathrm{DO}_{0}^{*}$ by working with de Rham cohomology only. The questions of whether $\Psi \mathrm{DO}_{0}^{*}$ is a tame Fréchet space [8, [13 and so has a good exponential map, the relationships among the cohomology with respect to the norm topology, the Fréchet topology and intermediate Banach norm topologies, and whether the de Rham theorem holds for $B \Psi \mathrm{DO}_{0}^{*}[\mathbf{2}$ are not addressed.

The role of $\Psi \mathrm{DO}_{0}^{*}$ in infinite dimensional geometry was explained to us by Sylvie Paycha, and we gratefully acknowledge our many conversations with her. We also would like to thank Varghese Mathai for suggesting we consider the closure of $\Psi \mathrm{DO}_{0}^{*}$ discussed below. The referee both pointed out serious errors and gave valuable suggestions for simplifying and clarifying this paper, which we gratefully acknowledge.

Finally, this paper is in many ways inspired by the seminal text [17 of Misha Shubin, whose clear writing has made a difficult subject accessible to so many mathematicians.

\section{Vanishing of Wodzicki-Chern classes of $\Psi D O-b u n d l e s$}

Let $\Psi \mathrm{DO}_{0}^{*}=\Psi \mathrm{DO}_{0}^{*}(E)$ be the group of zeroth order invertible classical $\Psi \mathrm{DO}$ acting on sections of a fixed finite rank complex bundle $E \rightarrow N$. We recall the setup for $\Psi \mathrm{DO}_{0}^{*}$-bundles. Fix a complete Riemannian metric on $N$ and a Hermitian metric 
on $E$. For a real parameter $s_{0} \gg 0$, let $H^{s_{0}} \Gamma(E)=H^{s_{0}} \Gamma(E \rightarrow N)$ be the sections of $E$ of Sobolev class $s_{0}$. This space depends on the Riemannian metric if $N$ is noncompact, and of course can be defined via local charts without choosing a metric. Let $\mathcal{E}$ be a Banach bundle over a base $B$ such that locally $\left.\mathcal{E}\right|_{U} \simeq U \times H^{s_{0}} \Gamma(E)$ and such that the transition functions lie in $\Psi \mathrm{DO}_{0}^{*}(E)$. Then we call $\mathcal{E}$ a $\Psi \mathrm{DO}_{0^{-}}^{*}$ or $\Psi \mathrm{DO}_{0}^{*}(E)$-bundle over $B$.

The role of $s_{0}$ is not very important. We could take the $C^{\infty}$ Fréchet topology on the sections of $E$, since this is a tame Fréchet space in the sense of $\mathbf{8}$.

As explained in [6], the tangent bundle to $\operatorname{Maps}^{s_{0}}(N, M)$, the space of $H^{s_{0}}$ maps between manifolds $N, M$, is a $\Psi \mathrm{DO}_{0}^{*}$-bundles. Fix a component $\operatorname{Maps}_{f}^{s_{0}}(N, M)$ of a map $f: M \rightarrow N$. We can take $H^{s_{0}} \Gamma\left(f^{*} T M \rightarrow N\right)$ as the tangent space $T_{f} \operatorname{Maps}^{s_{0}}(N, M)=T_{f} \operatorname{Maps}_{f}^{s_{0}}(M, N)$. Exponentiating sufficiently short sections $X \in H^{s_{0}} \Gamma\left(f^{*} T M\right)$ via $n \mapsto \exp _{f(n), M} X_{n}$ gives a coordinate neighborhood of $f$ in $\operatorname{Maps}^{s_{0}}(N, M)$, making $\operatorname{Maps}^{s_{0}}(N, M)$ into a Banach manifold. The transition maps for $T \operatorname{Maps}^{s_{0}}(N, M)$ for nearby maps are of the form $d \exp _{f_{1}} \circ d \exp _{f}^{-1}$, which are easily seen to be isomorphic to gauge transformations of $f^{*} T M$. Since gauge transformations are invertible multiplication operators, $T \operatorname{Maps}^{s_{0}}(N, M)$ is a $\Psi \mathrm{DO}_{0^{-}}^{*}$ bundle, although at this point there is no need to pass from gauge bundles to $\Psi \mathrm{DO}_{0}^{*}$-bundles. Note that the gauge group depends on the component of $f$. In particular, for the loop space $L M=\operatorname{Maps}^{s_{0}}\left(S^{1}, M\right)$, each complexified tangent space $T_{\gamma} L M$ is $H^{s_{0}} \Gamma\left(S^{1} \times \mathbb{C}^{n} \rightarrow S^{1}\right)$ for $M^{n}$ oriented. For convenience, we will always complexify tangent bundles.

Remark 2.1. These bundles fit into the framework of the families index theorem. Start with a fibration of manifolds with an auxiliary bundle

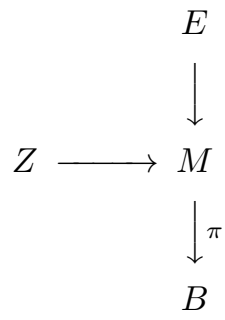

Here $M$ and $B$ are manifolds, and the fiber is modeled on a compact manifold $Z$. The structure group of the fibration is $\operatorname{Diff}(Z)$. Pushing down the sheaf of sections of $E$ via $\pi$ gives an infinite rank bundle

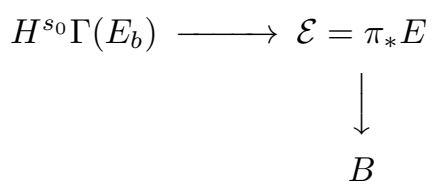

with the fiber modeled on the $H^{s_{0}}$ sections of $E_{b}=\left.E\right|_{Z_{b}}$ over $Z_{b}=\pi^{-1}(b)$ for one $b$ in each component of $B$. The structure group of $\mathcal{E}$ is now a semidirect product $\mathcal{G} \ltimes \operatorname{Diff}(Z)$, where $\mathcal{G}$ is the gauge group of $E_{b}$. In particular, for ev : 


$$
\begin{gathered}
N \times \operatorname{Maps}^{s_{0}}(N, M) \rightarrow M, \operatorname{ev}(n, f)=f(n), \text { and } \\
E=\operatorname{ev}^{*} T M \\
N \longrightarrow T M \\
\downarrow N \times \operatorname{Maps}^{s_{0}}(N, M) \stackrel{\text { ev }}{\perp} M \\
\operatorname{Maps}^{s_{0}}(N, M)
\end{gathered}
$$

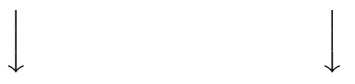

we get $\mathcal{E}=\pi_{*} \mathrm{ev}^{*} T M=T \operatorname{Maps}^{s_{0}}(N, M)$. Since the fibration is trivial, the structure group is just the gauge group. Defining characteristic classes for nontrivial fibrations is open at present.

As explained in the introduction, any trace on the gauge group $\mathcal{G}$ of $E \rightarrow N$ will give a Chern-Weil theory of characteristic classes on $\mathcal{E}$. Such a trace will be used in $\S 3$. However, using a wider class of traces is natural, as we now explain. The choice of Riemannian metrics on $N, M$ leads to a family of Riemannian metrics on $\operatorname{Maps}(N, M)$. Namely, pick $s \gg 0, s \leq s_{0}$. For $X, Y \in T_{f} \operatorname{Maps}^{s_{0}}(N, M)$, set

$$
\langle X, Y\rangle_{f, s}=\int_{N}\left\langle X_{n},(1+\Delta)^{s} Y_{n}\right\rangle_{f}(n) \operatorname{dvol}(n),
$$

where $\Delta=\left(\mathrm{ev}^{*} \nabla\right)^{*}\left(\mathrm{ev}^{*} \nabla\right)$ and $\nabla=\nabla^{M}$ is the Levi-Civita connection on $M$. Here we assume $N$ is compact. For example, when $N=S^{1}$, ev* $\nabla$ is covariant differentiation along the loop $f$. Equivalently, we are taking the $L^{2}$ inner product of the pointwise $H^{s}$ norms of $X$ and $Y$.

The metric (2.1) gives rise to a Levi-Civita connection $\nabla^{s}$ by the Koszul formula

$$
\begin{aligned}
\left\langle\nabla_{Y}^{s} X, Z\right\rangle_{s}=\quad X\langle Y, Z\rangle_{s}+Y\langle X, Z\rangle_{s}-Z\langle X, Y\rangle_{s} \\
\quad+\langle[X, Y], Z\rangle_{s}+\langle[Z, X], Y\rangle_{s}-\langle[Y, Z], X\rangle_{s} .
\end{aligned}
$$

provided the right hand side is a continuous linear functional of $Z \in$

$T_{f} \operatorname{Maps}^{s_{0}}(N, M)$. As explained in [12, the only problematic term $Z\langle X, Y\rangle_{s}$ is continuous in $Z$ for $s \in \mathbb{Z}^{+}$, but this probably fails otherwise.

Restricting ourselves to $s \in \mathbb{Z}^{+}$, we find that the connection one-form and curvature two-form of $\nabla^{s}$ take values in $\Psi \mathrm{DO}_{\leq 0}\left(\mathrm{ev}^{*} T M\right)$. (This is 12, Thm. 2.1, Prop. 2.3] for $L M$, and the proof generalizes.) Because these natural connections do not take values in $\Gamma \operatorname{End}(E, E)$, the Lie algebra of the gauge group $\operatorname{Aut}(E)$, we have to extend the structure group of $T \operatorname{Maps}^{s_{0}}(N, M)$ to $\Psi D_{0}^{*}$. Note that $\Psi D O_{0}^{*}$ acts as bounded operators on $T_{f} \operatorname{Maps}^{s_{0}}(N, M)$ for all choices of $s_{0}$, so the structure group is independent of this choice. The zeroth order parts of the connection and curvature forms are just the connection and curvature forms of $\mathrm{ev}^{*} \nabla_{f(n)}^{M}$, so only the negative order parts contain new information.

To extract the new information, we pick the unique trace on $\Psi \mathrm{DO}_{\leq 0}(E \rightarrow N)$ that detects negative order terms, namely the Wodzicki residue

$$
\operatorname{res}^{\mathrm{w}}: \Psi \mathrm{DO}_{\leq 0} \rightarrow \mathbb{C}, \operatorname{res}^{\mathrm{w}}(A)=(2 \pi)^{-n} \int_{S^{*} N} \operatorname{tr} \sigma_{-n}(A)(x, \xi) d \xi \operatorname{dvol}(x),
$$

where $S^{*} N$ is the unit cosphere bundle of $N^{n}$.

We now pass to general $\Psi \mathrm{DO}_{0}^{*}$-bundles $\mathcal{E} \rightarrow \mathcal{M}$ which admit connections; for example, if $\mathcal{M}$ admits a partition of unity, then $\mathcal{E} \rightarrow \mathcal{M}$ possesses connections. Standard Chern-Weil theory extends to justify the following definition. 
Definition 2.2. The $\mathrm{k}^{\text {th }}$ Wodzicki-Chern class $c_{k}^{\mathrm{w}}(\mathcal{E})$ of the $\Psi \mathrm{DO}_{0}^{*}$-bundle $\mathcal{E} \rightarrow \mathcal{M}$ admitting a connection $\nabla$ is the de Rham cohomology class $\left[\operatorname{res}^{\mathrm{w}}\left(\Omega^{k}\right)\right]$, where $\Omega$ is the curvature of $\nabla$.

As for $\operatorname{Maps}^{s_{0}}(N, M)$, the Wodzicki-Chern classes are independent of the choice of Sobolev parameter $s_{0}$. These classes easily vanish for $\Psi \mathrm{DO}_{0}^{*}$-bundles such as $T \operatorname{Maps}^{s_{0}}(N, M)$ which restrict to gauge bundles. For such bundles admit a connection taking values in the Lie algebra $\Gamma \operatorname{End}(E, E)$ of the gauge group. The curvature two-form is thus a multiplication operator with vanishing Wodzicki residue. Since the Wodzicki-Chern class is independent of connection, these classes vanish.

We give a subclass of $\Psi \mathrm{DO}_{0}^{*}$-bundles for which the Wodzicki-Chern classes vanish. Recall that paracompact Hilbert manifolds admit partitions of unity. Since $\operatorname{Maps}^{s_{0}}(N, M)$ is a Hilbert manifold and a metrizable space for $M, N$ closed, it is paracompact and so admits partitions of unity. Moreover, by a theorem of Milnor, $\operatorname{Maps}^{s_{0}}(N, M)$ has the homotopy type of a CW complex in the compact-open topology. This carries over to the Sobolev topology by e.g. putting a connection $\nabla$ on $M$, and using the heat operator associated to $\nabla^{*} \nabla$ to homotop continuous maps to smooth maps.

Theorem 2.3. Let Ell $^{*} \subset \Psi \mathrm{DO}_{0}^{*}$ be the subgroup of invertible zeroth order elliptic operators whose leading order symbol is the identity. Assume $\mathcal{M}$ is a manifold homotopy equivalent to a $C W$ complex and admitting a cover with a subordinate partition of unity. If $\mathcal{E} \rightarrow \mathcal{M}$ is an $\mathrm{Ell}^{*}$-bundle, then the Wodzicki-Chern classes $c_{k}^{\mathrm{w}}(\mathcal{E})$ are zero.

Proof. By [4, Prop. 15.4], for $V=S^{n}$ or $B^{n}$, a continuous map $f: V \rightarrow$ Ell $^{*}$ is homotopic within Ell* to a map $g: V \rightarrow \mathrm{GL}_{\infty}$, the set of operators of the form $I+P$, where $P$ is a finite rank operator. For $V=S^{n}$, we get that the inclusion $i: \mathrm{GL}_{\infty} \rightarrow \mathrm{Ell}^{*}$ induces a surjection $i_{*}: \pi_{k}\left(\mathrm{GL}_{\infty}\right) \rightarrow \pi_{k}\left(\mathrm{Ell}^{*}\right)$ on all homotopy groups, and for $V=B^{n}$ we get that $i_{*}$ is injective. From the diagram

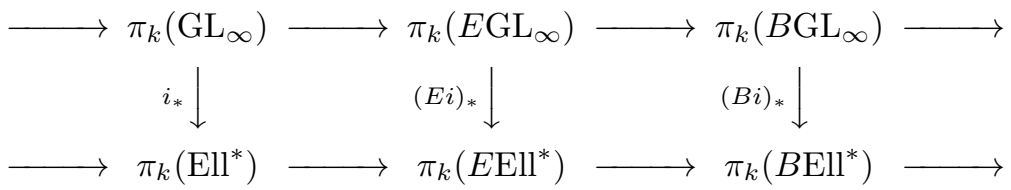

we get $(B i)_{*}: \pi_{k}\left(B \mathrm{GL}_{\infty}\right) \rightarrow \pi_{k}\left(B \mathrm{Ell}^{*}\right)$ is an isomorphism for all $k$. These classifying spaces are weakly equivalent to $\mathrm{CW}$ complexes [18, Thm. 7.8.1]. This implies that $\left[X, B \mathrm{GL}_{\infty}\right]=\left[X, B \mathrm{Ell}^{*}\right]$ for any $\mathrm{CW}$ complex $X$. In particular, any Ell*-bundle reduces to a $\mathrm{GL}_{\infty}$-bundle.

Thus $\mathcal{E} \rightarrow \mathcal{M}$ admits a $\mathrm{GL}_{\infty}$-connection. In fact, the proof of [4, Prop. 15.4] implicitly shows that $\mathrm{GL}_{\infty}$ is homotopy equivalent to $\Psi_{-\infty}^{*}$, the group of invertible $\Psi$ DOs of the form $I+P$, where $P$ is a finite rank operator given by a smooth kernel. Thus we may assume that the connection one-form $\theta$ takes values in $\operatorname{Lie}\left(\Psi_{-\infty}^{*}\right)$, the space of smoothing operators. The curvature two-form is given locally by $\Omega_{\alpha}=d \theta_{\alpha}+\theta_{\alpha} \wedge \theta_{\alpha}$, and hence $\Omega^{k}$ also takes values in smoothing operators. The Wodzicki residue of $\Omega^{k}$ therefore vanishes, so $c_{k}^{\mathrm{w}}(\mathcal{E})=0$.

Based on this result and calculations, the following conjecture seems plausible.

Conjecture 2.4. The Wodzicki-Chern classes vanish on any $\Psi \mathrm{DO}_{0}^{*}$-bundle over a base manifold admitting a partition of unity. 
We will see in $\S 3$ that $\mathrm{Ell}^{*}$ is not a deformation retraction of $\Psi \mathrm{DO}_{0}^{*}$ in general, so the conjecture does not follow from the previous theorem.

We now outline a putative proof of the conjecture based on the families index theorem setup for trivial fibrations as in Remark 2.1; details will appear elsewhere. As noted above, the Wodzicki-Chern classes vanish for these gauge bundles, but the superconnection techniques given below may generalize to other, perhaps all, $\Psi \mathrm{DO}_{0}^{*}$-bundles.

Let $\nabla=\nabla^{0} \oplus \nabla^{1}$ be a graded connection on a graded infinite dimensional bundle $\mathcal{E}=\mathcal{E}^{0} \oplus \mathcal{E}^{1}$ over a base space $B$. Let $R=R^{0} \oplus R^{1}$ be the corresponding curvature form. The connection and curvature forms take values in $\Psi$ DOs of nonpositive order.

We choose a smooth family of nonpositive order $\Psi$ DOs $a: \mathcal{E}^{0} \rightarrow \mathcal{E}^{1}$, and set

$$
A: \mathcal{E} \rightarrow \mathcal{E}, A=\left(\begin{array}{cc}
0 & a^{*} \\
a & 0
\end{array}\right)
$$

We form the superconnection $B_{t}=\nabla+t^{1 / 2} A$. For convenience, assume that $A$ has constant order zero. Then the heat operator $\exp \left(-B_{t}^{2}\right)$ is a smooth family of zero order $\Psi$ DOs, as seen from an analysis of its asymptotic expansion.

The standard transgression formula for the local families index theorem is of the form $\operatorname{str}\left(\exp \left(-B_{t_{1}}^{2}\right)\right)-\operatorname{str}\left(\exp \left(-B_{t_{2}}^{2}\right)\right)=d \int_{t_{1}}^{t_{2}} \alpha_{t} d t$, where str is the supertrace and $\int \alpha_{t}$ is an explicit Chern-Simons form [3. In the $t \rightarrow \infty$ limit, the connection becomes a connection on the finite rank index bundle, and defines there a smoothing operator on which the Wodzicki residue trace is zero. As $t \rightarrow 0$, the Wodzicki residue of $e^{-B_{t}^{2}}$ approaches the residue of $e^{-R}$. This limit exists because we are using the Wodzicki residue and not the classical trace, as again follows from an analysis of the symbol asymptotics. This shows that the Wodzicki-Chern character and hence the $c_{k}^{\mathrm{w}}(\mathcal{E})$ vanish in cohomology.

We can manipulate the choice of $A$ and the bundle $\mathcal{E}^{1}$ to infer the result for a non-graded bundle. That is, we take $\mathcal{E}^{0}, \nabla^{0}, R^{0}$ to be a fixed $\Psi \mathrm{DO}_{0}^{*}$-bundle with connection and curvature form, and take $\mathcal{E}^{1}$ to be a trivial bundle with a flat connection $\nabla^{1}$. Choose $a$ to be an elliptic family of $\Psi$ DOs of order zero parametrized by $B$. Then the graded Wodzicki-Chern character reduces to the Wodzicki-Chern character of $E^{0}$, and we are done.

It may be that a refined version of this argument gives the vanishing of the Wodzicki-Chern character as a differential form, in which the Wodzicki-ChernSimons classes of $\mathbf{1 2}$ would always be defined.

\section{Extending the leading order symbol trace}

In contrast to the Wodzicki-Chern classes, the leading order Chern classes are often nonzero, and we can use them to detect elements of $H^{*}\left(B \Psi \mathrm{DO}_{0}^{*}, \mathbb{C}\right)$. As above, let $\mathcal{E}$ be a $\Psi \mathrm{DO}_{0}^{*}$-bundle with fiber modeled on $H^{s_{0}} \Gamma(E \rightarrow N)$.

Throughout this section, we use the following conventions: (i) the manifold $N$ is closed and connected; (ii) all cohomology groups $H^{*}(X, \mathbb{C})$ are de Rham cohomology; (iii) $\operatorname{Maps}(N, M)$ denotes $\operatorname{Maps}^{s_{0}}(N, M)$ for a fixed large Sobolev parameter $s_{0}$; (iv) smooth sections of a bundle $F$ are denoted by $\Gamma F$. 
Definition 3.1. The $\mathrm{k}^{\text {th }}$ leading order Chern class $c_{k}^{\text {lo }}(\mathcal{E})$ of the $\Psi \mathrm{DO}_{0}^{*}$-bundle $\mathcal{E} \rightarrow \mathcal{M}$ admitting a connection $\nabla$ is the de Rham cohomology class of

$$
\int_{S^{*} N} \operatorname{tr} \sigma_{0}\left(\Omega^{k}\right)(n, \xi) d \xi \operatorname{dvol}(n)
$$

where $\Omega$ is the curvature of $\nabla$.

The point is that the leading order symbol trace $\int_{S^{*} N} \operatorname{tr} \sigma_{0}: \Psi \mathrm{DO}_{\leq 0} \rightarrow \mathbb{C}$ is a trace on this subalgebra, although it does not extend to a trace on all $\Psi$ DOs.

An obvious approach to calculating leading order classes would be to find a universal connection on $E \Psi \mathrm{DO}_{0}^{*} \rightarrow B \Psi \mathrm{DO}_{0}^{*}$. However, it seems difficult to build a model of $B \Psi \mathrm{DO}_{0}^{*}$ more concrete than the general Milnor construction. In particular, it is not clear that $B \Psi D O_{0}^{*}$ is a manifold, so the existence of a connection on $E \Psi \mathrm{DO}_{0}^{*}$ may be moot.

Alternatively, since elements of $\Psi \mathrm{DO}_{0}^{*}$ are bounded operators on the Hilbert space $\mathcal{H}=H^{s_{0}} \Gamma(E \rightarrow N)$, we can let $\bar{\Psi}=\overline{\Psi \mathrm{DO}_{0}^{*}}$ be the closure of $\Psi \mathrm{DO}_{0}^{*}$ in $G L(\mathcal{H})$ in the norm topology. $\bar{\Psi}$ acts freely on the contractible space $G L(\mathcal{H})$, so $E \bar{\Psi}=G L(\mathcal{H})$ and $B \bar{\Psi}=E \bar{\Psi} / \bar{\Psi} . G L(\mathcal{H})$ is a Banach manifold, and since the Frobenius theorem holds in this context, $B \bar{\Psi}$ is also a Banach manifold [13. In particular, $E \bar{\Psi} \rightarrow B \bar{\Psi}$ admits a connection. (It would be interesting to know if $E \bar{\Psi}$ has a universal connection.) Unfortunately, it is not clear that the leading order symbol trace extends to $\operatorname{Lie}(\bar{\Psi})$, so defining leading order symbol classes for $\bar{\Psi}$-bundles is problematic.

We separate these problems into two issues. The first strictly analytic issue is to find a large subalgebra of $\mathfrak{g l}(\mathcal{H})$ with an extension of the leading order symbol trace. Our solution in Proposition 3.6 in fact acts on a quotient algebra of a subalgebra of $\mathfrak{g l}(\mathcal{H})$. This leads to a different version of $\bar{\Psi}$ such that $\bar{\Psi}$-bundles with connection have a good theory of characteristic classes (see Definition 3.7). However, the existence of a universal $\bar{\Psi}$-bundle with connection is unclear, so we cannot use this theory to detect elements in $H^{*}\left(B \Psi \mathrm{DO}_{0}^{*}, \mathbb{C}\right)$.

The second issue is to find a Lie algebra $\mathfrak{g}$ such that $\Psi \mathrm{DO}_{\leq 0}$ surjects onto $\mathfrak{g}$ and such that the corresponding classifying space $B G$ is a manifold. In fact, we can reinterpret well known results to show that $\mathfrak{g}=H^{s_{0}} \Gamma \operatorname{End}\left(\pi^{*} E\right)$ works for $E \stackrel{\pi}{\rightarrow} N$. Since the leading order symbol trace extends to $\mathfrak{g}$, we can define characteristic classes of $\Psi D_{0}^{*}$-bundles to be pullbacks of the leading order symbol classes of $E G \rightarrow B G$ (Definition 4.1). This approach allows us to detect elements in $H^{*}\left(B \Psi \mathrm{DO}_{0}^{*}, \mathbb{C}\right)$ (Theorem 4.10).

In this section, we discuss analytic questions related to extensions of the leading order symbol trace. In $\S 4$, we discuss the topological questions related to the second issue.

To begin the analysis of the first issue, we first check that at the Lie algebra level, $\Psi \mathrm{DO}_{\leq 0}$ embeds continuously in $\mathfrak{g l}(\mathcal{H})$, a result probably already known. For a fixed choice of a finite precompact cover $\left\{U_{\ell}\right\}$ of $N$ and a subordinate partition of unity $\left\{\phi_{\ell}\right\}$, we write $A \in \Psi \mathrm{DO}_{\leq 0}$ as $A=A^{1}+A^{0}$, where $A^{1}=\sum_{j, k}^{\prime} \phi_{j} A \phi_{k}$, with the sum over $j, k$ with supp $\phi_{j} \cap \operatorname{supp} \phi_{k} \neq \emptyset$, and $A^{0}=A-A^{1}$. Then $A^{1}$ is properly supported and has the classical local symbol $\sigma\left(\phi_{j} A \phi_{k}\right)$ in $U_{j}$, and $A^{0}$ has a smooth kernel $k(x, y)$ [9. Prop. 18.1.22]. The Fréchet topology on the classical 
IDOs of nonpositive integer order is given locally by the family of seminorms

$$
\begin{gathered}
\sup _{x, \xi}\left|\partial_{x}^{\beta} \partial_{\xi}^{\alpha} \sigma\left(\phi_{j} A \phi_{k}\right)(x, \xi)\right|(1+|\xi|)^{|\alpha|}, \\
\sup _{x,|\xi|=1}\left|\partial_{x}^{\beta} \partial_{\xi}^{\alpha} \sigma_{-m}\left(\phi_{j} A \phi_{k}\right)(x, \xi)\right|, \\
\sup _{x, \xi}\left|\partial_{x}^{\beta} \partial_{\xi}^{\alpha}\left(\sigma\left(\phi_{j} A \phi_{k}\right)(x, \xi)-\psi(\xi) \sum_{m=0}^{T-1} \sigma_{-m}\left(\phi_{j} A \phi_{k}\right)(x, \xi)\right)\right|(1+|\xi|)^{|\alpha|+T}, \\
\sup _{x, y}\left|\partial_{x}^{\alpha} \partial_{y}^{\beta} k(x, y)\right|,
\end{gathered}
$$

where $\psi$ is a smooth function vanishing near zero and identically one outside a small ball centered at the origin [9, §18.1]. The topology is independent of the choices of $\psi,\left\{U_{\ell}\right\}$, and $\left\{\phi_{\ell}\right\}$. Since elements $A$ of the gauge group $\mathcal{G}$ of $E$ are order zero multiplication operators with $\sigma_{0}(A)(x, \xi)$ independent of $\xi$, the gauge group inherits the usual $C^{\infty}$ Fréchet topology in $x$.

Lemma 3.2. For the Fréchet topology on $\Psi \mathrm{DO}_{\leq 0}$ and the norm topology on $\mathfrak{g l}(\mathcal{H})$, the inclusion $\Psi \mathrm{DO}_{\leq 0} \rightarrow \mathfrak{g l}(\mathcal{H})$ is continuous.

Proof. We follow [7, Lemma 1.2.1]. Let $A_{i} \rightarrow 0$ in $\Psi D O_{\leq 0}$. Since $H^{s_{0}}$ is isometric to $L^{2}=H^{0}$ for any $s_{0}$, it suffices to show that $\left\|A_{i}\right\| \rightarrow 0$ for $A_{i}: L^{2} \rightarrow L^{2}$.

As usual, the computations reduce to estimates in local charts. We abuse notation by writing $\sigma\left(\phi_{j} A_{i} \phi_{k}\right)$ as $\sigma\left(A_{i}^{1}\right)=a_{i}$. Then

$$
\widehat{A_{i}^{1} f}(\zeta)=\int e^{i x \cdot(\xi-\zeta)} a_{i}(x, \xi) \hat{f}(\xi) d \xi d x=\int q_{i}(\zeta-\xi, \xi) \hat{f}(\xi) d \xi
$$

for $q_{i}(\zeta, \xi)=\int e^{-i x \cdot \zeta} a_{i}(x, \xi) d x$. (We are using a normalized version of $d x$ in the Fourier transform.) By [7, Lemma 1.1.6], $\left|A_{i}^{1} f\right|_{0}=\sup _{g} \frac{\left|\left(A_{i}^{1} f, g\right)\right|}{|g|_{0}}$, where $g$ is a Schwarz function and we use the $L^{2}$ inner product. By Cauchy-Schwarz,

$$
\left|\left(A_{i}^{1} f, g\right)\right| \leq\left(\int\left|q_{i}(\zeta-\xi, \xi)\right||\hat{f}(\xi)|^{2} d \zeta d \xi\right)^{1 / 2}\left(\int\left|q_{i}(\zeta-\xi, \xi)\right||\hat{g}(\xi)|^{2} d \zeta d \xi\right)^{1 / 2} .
$$

We claim that

$$
\left|q_{i}(\zeta-\xi, \xi)\right| \leq C_{i}, \int\left|q_{i}(\zeta-\xi, \xi)\right| d \xi \leq C_{i}^{\prime}, \int\left|q_{i}(\zeta-\xi, \xi)\right| d \zeta \leq C_{i}^{\prime \prime},
$$

with $C_{i}, C_{i}^{\prime}, C_{i}^{\prime \prime} \rightarrow 0$ as $i \rightarrow \infty$. If so, $\left|\left(A_{i}^{1} f, g\right)\right| \leq D_{i}|f|_{0}|g|_{0}$ with $D_{i} \rightarrow 0$, and so $\left\|A_{i}^{0}\right\| \rightarrow 0$.

For the claim, we know $\left|\partial_{x}^{\alpha} a_{i}(x, \xi)\right| \leq C_{\alpha, i}$ with $C_{\alpha, i} \rightarrow 0$. Since $a_{i}$ has compact $x$ support, we get

$$
\left|\zeta^{\alpha} q_{i}(\zeta, \xi)\right|=\left|\int e^{-i x \cdot \zeta} \partial_{x}^{\alpha} a_{i}(x, \xi) d x\right| \leq C_{\alpha, i}
$$

for a different constant decreasing to zero. In particular, $\left|q_{i}(\zeta, \xi)\right| \leq C_{i}(1+$ $|\xi|)^{-1-n / 2}$, for $n=\operatorname{dim}(N)$. This shows that $q_{i}(\zeta-\xi, \xi)$ is integrable and satisfies (3.2).

It is straightforward to show that $A_{i}^{0} \rightarrow 0$ in the Fréchet topology on smooth kernels implies $\left\|A_{i}^{0}\right\| \rightarrow 0$. Thus $\left\|A_{i}\right\| \leq\left\|A_{i}^{0}\right\|+\left\|A_{i}^{1}\right\| \rightarrow 0$. 
In order to extend the leading order Chern class to $\bar{\Psi}$-bundles, we associate an operator to the symbol of $A \in \Psi D_{\leq 0}$. Set

$$
\mathrm{Op}_{1}(A)(f)(x)=\sum_{j, k}^{\prime} \int_{U_{j}} e^{i(x-y) \cdot \xi} \sigma\left(\phi_{j} A \phi_{k}\right)(x \cdot \xi) f(y) d y d \xi
$$

Then $A-\mathrm{Op}_{1}(A) \in \Psi \mathrm{DO}_{-\infty}$, the closed ideal of $\Psi$ DOs of order $-\infty$, and $\sigma(A) \stackrel{\text { def }}{=}$ $\sigma\left(A^{1}\right)=\sigma\left(\mathrm{Op}_{1}(A)\right)$. Note that $\mathrm{Op}_{1}(A)$ is shorthand for the $\Psi \mathrm{DO} \operatorname{Op}(\sigma(A))$ noncanonically associated to $\sigma(A) \in \Gamma\left(\operatorname{End}\left(\pi^{*} E\right) \rightarrow S^{*} N\right)$.

\section{Definition 3.3.}

$$
\mathrm{Op}_{1}=\left\{\mathrm{Op}_{1}(A): A \in \Psi \mathrm{DO}_{\leq 0}\right\}
$$

We emphasize that $\mathrm{Op}_{1}$ depends on a fixed atlas and subordinate partition of unity for $N$. The closed vector space $\mathrm{Op}_{1}$ is not an algebra, but the linear map $o: \Psi \mathrm{DO}_{\leq 0} \rightarrow \mathrm{Op}_{1}, A \mapsto \mathrm{Op}_{1}(A)$ is continuous. Let $\overline{\mathrm{Op}}_{1}$ be the closure of $\mathrm{Op}_{1}$ in $\mathfrak{g} l(\mathcal{H})$.

Fix $K>0$, and set

$$
\begin{aligned}
\mathrm{Op}_{1}^{K}= & \left\{\mathrm{Op}_{1}(A):\left|\partial_{\xi}^{\alpha}\left(\sigma(A)-\sigma_{0}(A)\right)(x, \xi)\right| \leq K(1+|\xi|)^{-1},\right. \\
& \left.\left|\partial_{\xi}^{\alpha} \sigma_{0}(A)(x, \xi)\right| \leq K, \text { for }|\alpha| \leq 1, \forall(x, \xi) \in T^{*} U_{j}, \forall j\right\} .
\end{aligned}
$$

Since $\sigma_{0}$ has homogeneity zero and $S^{*} N$ is compact, every $\mathrm{Op}_{1}(A) \in \mathrm{Op}_{1}$ lies in some $\mathrm{Op}_{1}^{K}$.

Lemma 3.4. $A \mapsto \int_{S^{*} N} \operatorname{tr} \sigma_{0}(A)(n, \xi) d \xi \operatorname{dvol}(n)$ extends from a continuous map on $\mathrm{Op}_{1}^{K}$ to a continuous map on $\overline{\mathrm{Op}_{1}^{K}}$.

Proof. We must show that if $\left\{\mathrm{Op}_{1}\left(A_{i}\right)\right\} \subset \mathrm{Op}_{1}^{K}$ is Cauchy in the norm topology on $\operatorname{End}(\mathcal{H})$, then $\left\{\operatorname{tr} \sigma\left(A_{i}\right)\right\}$ is Cauchy in $L^{1}\left(S^{*} N\right)$. Fix a finite cover $\left\{U_{\ell}\right\}$ of $N$ with $\bar{U}_{\ell}$ compact. The hypothesis is

$$
\left\|\int_{T^{*} U_{k}} e^{i n \cdot \xi} \phi_{\ell}(n)\left(\sigma\left(A_{i}\right)-\sigma\left(A_{j}\right)\right)(n, \xi) \phi_{k}(n) \widehat{f}(\xi) d \xi\right\|_{s_{0}}<\epsilon\|f\|_{s_{0}}
$$

for $i, j>N(\epsilon)$, and for each $\ell, k$ with supp $\phi_{\ell} \cap \operatorname{supp} \phi_{k} \neq \emptyset$. Sobolev embedding implies that

$$
n \mapsto g_{i, j}(n)=\frac{1}{\|f\|_{s_{0}}} \int_{T_{n}^{*} U_{k}} e^{i n \cdot \xi} \phi_{\ell}(n)\left(\sigma\left(A_{i}\right)-\sigma\left(A_{j}\right)\right)(n, \xi) \phi_{k}(n) \widehat{f}(\xi) d \xi
$$

is $\epsilon$-small in $C^{r}\left(U_{k}\right)$ for any $r<s_{0}-(\operatorname{dim} N) / 2$ and any fixed $f \in H^{s_{0}}$.

Fix $U_{\ell}$, and pick $\xi_{0}$ in the cotangent space of a point $n_{1} \in U_{\ell}$ with $\phi_{\ell}\left(n_{1}\right) \phi_{k}\left(n_{1}\right)$ $\neq 0$. We can identify all cotangent spaces in $T^{*} U_{\ell}$ with $T_{n_{1}}^{*} U_{\ell}$. We claim that

$$
h_{i, j}\left(n, \xi_{0}\right)=\phi_{\ell}(n)\left(\sigma\left(A_{i}\right)-\sigma\left(A_{j}\right)\right)\left(n, \xi_{0}\right) \phi_{k}(n)
$$

has $\left|h_{i, j}\left(n, \xi_{0}\right)\right|<\epsilon$ for all $n \in U_{k}$, for all $\ell$, and for $i, j \gg 0$. Since $\sigma_{0}$ has homogeneity zero, we may assume that $\xi_{0} \in S^{*} N$. Thus we claim that $\left\{\phi_{\ell}(n) \sigma\left(A_{i}\right)\left(n, \xi_{0}\right) \phi_{k}(n)\right\}$ is Cauchy in this fixed chart, and so by compactness $\left\{\sum_{\ell, k}^{\prime} \sigma\left(\phi_{\ell} A_{i} \phi_{k}\right)=\sigma\left(A_{i}\right)\right\}$ will be uniformly Cauchy on all of $S^{*} N$.

For the moment, we assume that our symbols are scalar valued. If the claim fails, then by compactness of $N$ there exists $n_{0}$ and $\epsilon>0$ such that there exist $i_{k}, j_{k} \rightarrow \infty$ with $\left|h_{i_{k} j_{k}}\left(n_{0}, \xi_{0}\right)\right|>\epsilon$. Let $\widehat{f}=\widehat{f}_{\xi_{0}, \delta}$ be a bump function of height $e^{-i n_{0} \cdot \xi_{0}}$ concentrated on $B_{r(\delta)}\left(\xi_{0}\right)$, the metric ball in $T_{n_{0}}^{*} U_{\ell}$ centered at $\xi_{0}$ and of 
volume $\delta$, and let $b_{\xi_{0}, \delta}$ be the corresponding bump function of height one. Taylor's theorem in the form

$$
a\left(\xi_{0}\right)-a(\xi)=-\sum_{\ell}\left(\xi-\xi_{0}\right)^{k} \int_{0}^{1} \partial_{\xi}^{\ell} a\left(\xi_{0}+t\left(\xi-\xi_{0}\right)\right) d t
$$

applied to $a(\xi)=h_{i_{k} j_{k}}\left(n_{0}, \xi\right)$ implies

$$
\begin{aligned}
& \left|\int_{T_{n_{0}}^{*} U_{\ell}} e^{i n_{0}\left(\xi-\xi_{0}\right)} h_{i_{k} j_{k}}\left(n_{0}, \xi\right) b_{\xi_{0}, \delta} d \xi\right| \\
& \quad \geq\left|h_{i_{k} j_{k}}\left(n_{0}, \xi_{0}\right) \int_{T_{n_{0}}^{*} U_{\ell}} e^{i n_{0} \xi} b_{\xi_{0}, \delta} d \xi\right| \\
& \quad-\left|\int_{T_{n_{0}}^{*} U_{\ell}} e^{i n_{0}\left(\xi-\xi_{0}\right)}\left(h_{i_{k} j_{k}}\left(n_{0}, \xi_{0}\right)-h_{i_{k} j_{k}}\left(n_{0}, \xi\right)\right) b_{\xi_{0}, \delta} d \xi\right| \\
& \geq \frac{1}{2} h_{i_{k} j_{k}}\left(n_{0}, \xi_{0}\right) \delta-r(\delta) F\left(\delta,\left(n_{0}, \xi_{0}\right)\right) \delta
\end{aligned}
$$

for some $F\left(\delta,\left(n_{0}, \xi_{0}\right)\right) \rightarrow 0$ as $\delta \rightarrow 0$. To produce this $F$, we use $\left|\left(\xi-\xi_{0}\right)^{k}\right| \leq$ $r(\delta)$ and (3.3) with $|\alpha|=1$ to bound the partial derivatives of $h_{i_{k} j_{k}}\left(n_{0}, \xi\right)$ in (3.5) by a constant independent of $i_{k}, j_{k}$. For $\delta$ small enough, $\left.r(\delta) F\left(\delta,\left(n_{0}, \xi_{0}\right)\right)\right)<$ $\frac{1}{4}\left|h_{i_{k} j_{k}}\left(n_{0}, \xi_{0}\right)\right|$ for all $k$, and so

$$
\left|\int_{T_{n_{0}}^{*} U_{\ell}} e^{i n_{0}\left(\xi-\xi_{0}\right)} h_{i_{k} j_{k}}\left(n_{0}, \xi\right) b_{\xi_{0}, \delta} d \xi\right|>\frac{1}{4}\left|h_{i_{k} j_{k}}\left(n_{0}, \xi_{0}\right)\right| \delta
$$

Similarly, with some abuse of notation, we have

$$
\left.\|f\|_{s_{0}}=\left.\left(\sum_{\ell} \int_{U_{\ell}}\left(1+|\xi|^{2}\right)^{s_{0}} \mid \widehat{\left(\phi_{\ell} \cdot f\right.}\right)(\xi)\right|^{2} d \xi\right)^{1 / 2} .
$$

Since $\left.\mid \widehat{\left(\phi_{\ell} \cdot f\right.}\right)(\xi) \mid \leq C \cdot \hat{\phi}_{\ell}\left(\xi-\xi_{0}\right) \delta$ for some constant $C$ which we can take independent of $\delta$ for small $\delta$, we get

$$
\begin{aligned}
\|f\|_{s_{0}} & \leq\left(\Sigma_{\ell} \int_{U_{\ell}}\left(1+|\xi|^{2}\right)^{s_{0}} C \delta^{2}\left|\hat{\phi}_{\ell}\left(\xi-\xi_{0}\right)\right|^{2}\right)^{1 / 2} \\
& \leq C \delta
\end{aligned}
$$

where $C$ changes from line to line.

Substituting (3.6) and (3.7) into (3.4), we obtain

$$
\left|g_{i_{k} j_{k}}\left(n_{0}\right)\right| \geq C\left|h_{i_{k} j_{k}}\left(n_{0}, \xi_{0}\right)\right| \geq C \epsilon
$$

for all $k$, a contradiction. Thus $h_{i j}\left(n, \xi_{0}\right)$ has the claimed estimate.

If the symbol is matrix valued, we replace the bump functions by sections of the bundle $E$ having the $r^{\text {th }}$ coordinate in some local chart given by the bump functions and all other coordinates zero. The argument above shows that the $r^{\text {th }}$ columns of $\sigma\left(A_{i}\right)$ form a Cauchy sequence, and so each sequence of entries $\left\{\sigma\left(A_{i}\right)_{r}^{s}\right\}$ is Cauchy. 
If $\left\{\operatorname{tr}\left(\sigma_{0}\left(A_{i}\right)\right)\right\}$ is not uniformly Cauchy on $S^{*} N$, then there exists $\epsilon>0$ with an infinite sequence of $i, j$ and $(n, \xi) \in S^{*} N$ such that

$$
\begin{aligned}
& \left|\operatorname{tr}\left(\sigma\left(A_{i}\right)\right)(n, \lambda \xi)-\operatorname{tr}\left(\sigma\left(A_{j}\right)\right)(n, \lambda \xi)\right| \\
& \left.\quad=\mid \operatorname{tr}\left(\left(\sigma-\sigma_{0}\right)\left(A_{i}\right)\right)(n, \lambda \xi)+\operatorname{tr} \sigma_{0}\left(A_{i}\right)\right)(n, \lambda \xi) \\
& \left.\quad-\operatorname{tr}\left(\left(\sigma-\sigma_{0}\right)\left(A_{j}\right)\right)(n, \lambda \xi)-\operatorname{tr} \sigma_{0}\left(A_{j}\right)\right)(n, \lambda \xi) \mid \\
& \left.\geq \quad \mid \operatorname{tr}\left(\sigma_{0}\left(A_{i}\right)\right)(n, \xi)-\operatorname{tr} \sigma_{0}\left(A_{j}\right)\right)(n, \xi) \mid \\
& \quad-\left|\operatorname{tr}\left(\left(\sigma-\sigma_{0}\right)\left(A_{i}\right)\right)(n, \lambda \xi)-\operatorname{tr}\left(\left(\sigma-\sigma_{0}\right)\left(A_{j}\right)\right)(n, \lambda \xi)\right| \\
& \geq \quad \epsilon-2 K(1+|\lambda|)^{-1}
\end{aligned}
$$

for all $\lambda>0$. For $\lambda \gg 0$ this contradicts that $h_{i, j}$ is Cauchy.

This implies that $\left\{\operatorname{tr}\left(\sigma_{0}\left(A_{i}\right)\right)\right\}$ is uniformly Cauchy on $S^{*} N$, so the claimed extension exists. The continuity of the extension is immediate.

Fix $K$, and set

$$
\mathcal{A}=\mathcal{A}^{K}=\cup_{n \in \mathbb{Z}^{+}} \overline{\mathrm{Op}_{1}^{n K}} \subset \mathfrak{g l}(\mathcal{H}),
$$

where the closure is taken in the norm topology. Then

$$
\overline{\mathcal{A}}=\overline{\cup_{n \in \mathbb{Z}^{+}} \overline{\mathrm{Op}_{1}^{n K}}}=\overline{\cup_{n \in \mathbb{Z}^{+}} \mathrm{Op}_{1}^{n K}}=\overline{\mathrm{Op}_{1}} \subset \mathfrak{g l}(\mathcal{H})
$$

is independent of $K$.

Corollary 3.5. $A \mapsto \int_{S^{*} N} \operatorname{tr} \sigma_{0}(A)(n, \xi) d \xi \operatorname{dvol}(n)$ extends from a continuous map on $\mathrm{Op}_{1}$ to a continuous map on $\overline{\mathrm{Op}_{1}}=\overline{\mathcal{A}}$.

Proof. Fix $K$. We first show that the leading order symbol extends to $\mathcal{A}$. For $n^{\prime}>n$, the inclusion $i_{n, n^{\prime}}: \overline{\mathrm{Op}_{1}^{n K}} \rightarrow \overline{\mathrm{Op}_{1}^{n^{\prime} K}}$ has $\sigma^{n^{\prime} K} \circ i_{n, n^{\prime}}=\sigma^{n K}$ for the extensions $\sigma^{n K}, \sigma^{n^{\prime} K}$ on $\overline{\mathrm{Op}_{1}^{n K}}, \overline{\mathrm{Op}_{1}^{n K^{\prime}}}$. Thus for any $K$, we can unambiguously set $\sigma(A)=\sigma^{n K}(A)$ for $A \in \overline{\mathrm{Op}_{1}^{n K}}$. The continuity follows from the previous lemma. Since the extension is linear, it is immediate that the extension is infinitely Fréchet differentiable on $\mathcal{A}$ inside the Banach space $\mathfrak{g} l(\mathcal{H})$.

Since $\sigma$ is continuous on $\mathcal{A}$, it extends to a continuous linear functional on $\overline{\mathcal{A}}$, which is again smooth.

To discuss the tracial properties of extensions of $\int_{S^{*} N} \operatorname{tr} \sigma_{0}$, we must have algebras of operators. Since $\mathrm{Op}_{1}(A B)-\mathrm{Op}_{1}(A) \mathrm{Op}_{1}(B) \in \Psi \mathrm{DO} \mathrm{O}_{-\infty}, \mathrm{Op}_{1} / \Psi \mathrm{DO} \mathrm{-}_{-\infty}$ (i.e. $\left.\mathrm{Op}_{1} /\left(\Psi \mathrm{DO} \mathrm{O}_{-\infty} \cap \mathrm{Op}_{1}\right)\right)$ is an algebra. Since $A-\mathrm{Op}_{1}(A) \in \Psi \mathrm{DO}_{-\infty}$, we have $\mathrm{Op}_{1} / \Psi \mathrm{DO} O_{-\infty} \simeq \Psi \mathrm{DO}_{\leq 0} / \Psi \mathrm{DO} \mathrm{-}_{-\infty}$. Note that if $\mathrm{Op}_{1}^{\prime}(A)$ is defined as for $\mathrm{Op}_{1}(A)$ but with respect to a different atlas and partition of unity, then $\mathrm{Op}_{1}(A)-\mathrm{Op}_{1}^{\prime}(A)$ is a smoothing operator. Thus $\mathrm{Op}_{1} / \Psi \mathrm{DO} \mathrm{-}_{-\infty}$ is canonically defined, independent of these choices.

Let $\overline{\mathrm{Op}}_{1}$ be the closure of $\mathrm{Op}_{1}$ in $\mathfrak{g l}(\mathcal{H})$, and let $\mathcal{C}$ denote the closure of $\Psi D \mathrm{D}_{-\infty}$ in $\overline{\mathrm{Op}}_{1}$. $\mathcal{C}$ is easily a closed ideal in $\overline{\mathrm{Op}}_{1}$. On quotients of normed algebras, we take the quotient norm $\|[A]\|=\inf \{\|A\|: A \in[A]\}$.

Proposition 3.6. $A \mapsto \int_{S^{*} N} \operatorname{tr} \sigma_{0}(A)(n, \xi) d \xi \operatorname{dvol}(n)$ extends from a continuous trace on $\Psi \mathrm{DO}_{\leq 0} / \Psi \mathrm{DO}_{-\infty}$ to a continuous trace $\sigma$ on $\overline{\mathrm{Op}}_{1} / \mathcal{C} \subset \mathfrak{g l}(\mathcal{H}) / \mathcal{C}$.

Proof. Since $\Psi D_{-\infty} \subset \mathcal{C}$, it is immediate that the leading order symbol integral descends to a continuous trace on $\Psi D O_{\leq 0} / \Psi D O_{-\infty} \simeq \mathrm{Op}_{1} / \Psi D O_{-\infty}$ and extends to a continuous map on $\overline{\mathrm{Op}}_{1} / \mathcal{C}$. To see that the extension is a trace, take 
$A, B \in \overline{\mathrm{Op}}_{1} / \mathcal{C}$ and $A_{i}, B_{i} \in \mathrm{Op}_{1} / \Psi \mathrm{DO}_{-\infty}$ with $A_{i} \rightarrow A, B_{i} \rightarrow B$. The leading order symbol trace vanishes on $\left[A_{i}, B_{i}\right]$, so by continuity $\sigma([A, B])=0$.

We can pass from the Lie algebra to the Lie group level via the commutative diagram

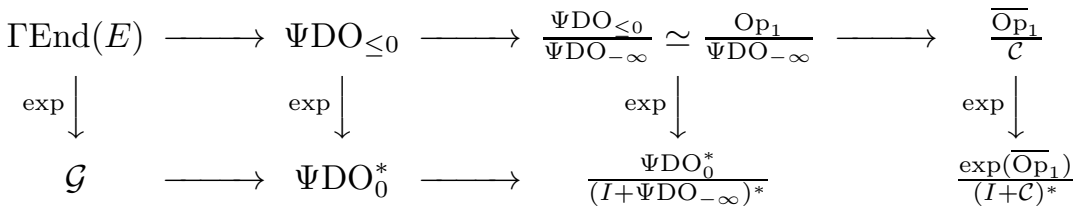

$\mathcal{G}$ is the gauge group of $E \stackrel{\pi}{\rightarrow} N,\left(I+\Psi \mathrm{DO}_{-\infty}\right)^{*}$ refers to invertible operators $I+B, B \in \Psi \mathrm{DO}_{-\infty}$, and similarly for other groups on the bottom line. The diagram consists of continuous maps if the spaces in the first three columns have either the norm or the Fréchet topology and the spaces in the last column have the norm topology. The exponential map is clearly surjective in the first column, and by standard Banach space arguments, the exponential map is surjective in the fourth column. The surjectivity of the second and third column is not obvious. In particular, there is no obvious map from $\frac{\Psi \mathrm{DO}_{0}^{*}}{\left(I+\Psi \mathrm{DO}_{-\infty}\right)^{*}}$ to $\frac{\exp \left(\overline{\mathrm{Op}}_{1}\right)}{(I+\mathcal{C})^{*}}$. In any case, the maps on the bottom line are group homomorphisms.

As discussed in the beginning of this section, it would be desirable to work with $\bar{\Psi}=\overline{\Psi \mathrm{DO}_{0}^{*}}$ or the closure of the standard variant $\frac{\Psi \mathrm{DO}_{0}^{*}}{\left(I+\Psi \mathrm{DO}_{-\infty}\right)^{*}}$. However, it seems difficult if not impossible to extend the leading order symbol trace to the corresponding Lie algebras $\overline{\Psi \mathrm{DO}_{\leq 0}}, \overline{\Psi \mathrm{DO}_{\leq 0}} / \overline{\Psi \mathrm{DO}_{-\infty}}$. The following re-definition is our substitute for $\overline{\Psi \mathrm{DO}_{0}^{*}}$, as it is the group associated to the largest known Lie algebra with an extension (strictly speaking, a factorization) of the leading order symbol trace.

Definition 3.7. $\bar{\Psi}=\frac{\exp \left(\overline{\mathrm{Op}}_{1}\right)}{(I+\mathcal{C})^{*}}$.

The smooth factorization of the leading order symbol trace $\sigma: \Psi \mathrm{DO}_{\leq 0} \rightarrow \mathbb{C}$ through $\overline{\mathrm{Op}}_{1} / \mathcal{C}$ gives us a geometric theory of characteristic classes for $\bar{\Psi}$-bundles.

Theorem 3.8. The leading order Chern classes $c_{k}^{\text {lo }}$ extend to $\bar{\Psi}$-bundles over paracompact manifolds.

Proof. Such bundles $\mathcal{E} \rightarrow B$ admit connections with curvature $\Omega \in$ $\Lambda^{2}\left(B, \overline{\mathrm{Op}}_{1} / \mathcal{C}\right)$. By the previous proposition, we may set

$$
c_{k}^{\text {lo }}(\nabla)_{b} \stackrel{\text { def }}{=} \sigma\left(\Omega_{b}^{k}\right)
$$

at each $b \in B$. Since $\sigma$ is smooth, the corresponding de Rham $c_{k}^{\text {lo }}(E \bar{\Psi})$ class is closed and independent of connection as in finite dimensions.

In summary, the leading order trace on $\Psi \mathrm{DO}_{0}^{*}$-bundles trivially factors through $\Psi \mathrm{DO}_{0}^{*} /\left(I+\Psi \mathrm{DO}_{-\infty}\right)^{*}$-bundles, as this quotient just removes the smoothing term $A^{0}$ from an invertible $\Psi$ DO $A$. By Proposition 3.6, this trace then extends continuously to $\overline{\mathrm{Op}}_{1} / \mathcal{C}$. This space is morally the closure of $\mathrm{Op}_{1} / \Psi \mathrm{DO}_{-\infty}$ in $\mathfrak{g l}(\mathcal{H}) / \mathcal{C}$, but it is not clear that $\mathcal{C}$ is an ideal in $\mathfrak{g l}(\mathcal{H})$. In any case, the work in this section has the feel of extending a continuous trace from a set to its closure. 
Remark 3.9. The groups in (3.8) give rise to different bundle theories, since the homotopy types of $\Psi \mathrm{DO}_{0}^{*}$, and $\Psi \mathrm{DO}_{0}^{*} /\left(I+\Psi \mathrm{DO}_{-\infty}\right)^{*}$ differ [16] the homotopy type of $\mathcal{G}$, discussed in [1], is almost surely different from that of $\Psi \mathrm{DO}_{0}^{*}, \Psi \mathrm{DO}_{0}^{*} /(I+$ $\left.\Psi \mathrm{DO}_{-\infty}\right)^{*}$. The relationship between the topology of $\Psi \mathrm{DO}_{0}^{*} /\left(I+\Psi \mathrm{DO}_{-\infty}\right)^{*}$ and $\bar{\Psi}=\exp \left(\overline{\mathrm{Op}}_{1}\right)(I+\mathcal{C})^{*}$ is completely open, so presumably the bundle theories for these groups also differ.

Geometrically, one can construct $\bar{\Psi}$-connections by using a partition of unity to glue together trivial connections over trivializing neighborhoods in a paracompact base. However, even for finite dimensional Lie groups, it is difficult to compute the corresponding Chern classes of such glued up connections. As a result, we do not have examples of nontrivial leading order Chern classes for $\bar{\Psi}$-bundles.

\section{Detecting cohomology of $B \Psi \mathrm{DO}_{0}^{*}$}

We now turn to the second issue discussed in the beginning of $\S 3$, namely that classifying spaces are not manifolds in general. We could obtain information about $H^{*}(B \bar{\Psi}, \mathbb{C})$ if $E \bar{\Psi} \rightarrow B \bar{\Psi}$ admitted a connection, but this presupposes that $B \bar{\Psi}$ is a manifold. As an alternative, we consider the exact sequence of algebras associated to $E \stackrel{\pi}{\rightarrow} N$ :

$$
0 \rightarrow \Psi \mathrm{DO}_{-1} \rightarrow \Psi \mathrm{DO}_{\leq 0} \stackrel{\sigma_{0}}{\rightarrow} \Gamma \operatorname{End}\left(\pi^{*} E \rightarrow S^{*} N\right) \rightarrow 0,
$$

which gives $\Gamma \operatorname{End}\left(\pi^{*} E \rightarrow S^{*} N\right) \simeq \Psi \mathrm{DO}_{\leq 0} / \Psi \mathrm{DO}_{-1}$. Here $\Psi \mathrm{DO}_{-1}$ is the algebra of classical integer order $\Psi D O$ s of order at most -1 . Note that the quotient $\Psi \mathrm{DO}_{\leq 0} / \Psi \mathrm{DO}_{-\infty}$ considered in $\S 3$ is more complicated topologically than $\Psi \mathrm{DO}_{\leq 0} / \Psi \mathrm{DO}_{-1}$.

We obtain the diagram

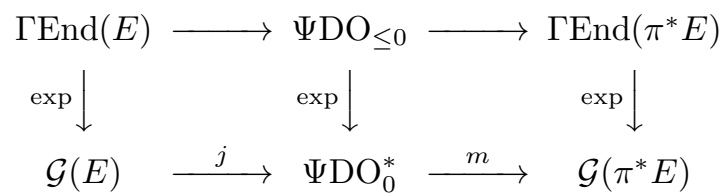

By Lemma 3.2 $j$ and $m$ are continuous, where $\mathcal{G}(E)$ has the Fréchet or norm topology, $\Psi \mathrm{DO}_{0}^{*}$ has the Frechét topology, and $\mathcal{G}\left(\pi^{*} E\right)$ has the Fréchet or the norm topology. The bottom line of this diagram induces

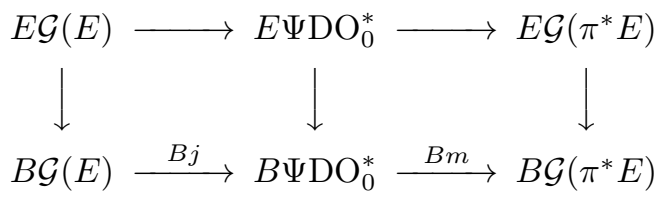

since $E \Psi \mathrm{DO}_{0}^{*} \simeq(B m)^{*} E \mathcal{G}\left(\pi^{*} E\right)$ and similarly for $E \mathcal{G}(E)$ by the Milnor construction.

We can now define leading order Chern classes of $\Psi \mathrm{DO}_{0}^{*}$-bundles, avoiding the question of the existence of connections on $E \Psi \mathrm{DO}_{0}^{*}$. By [1], for $E^{\ell} \rightarrow N$ and $\mathcal{G}=\mathcal{G}(E), B \mathcal{G}=\operatorname{Maps}_{0}(N, B U(\ell))=\left\{f: N \rightarrow B U(\ell) \mid f^{*} E U(\ell) \simeq E\right\}$, and $\left.E \mathcal{G}\right|_{f}$ is the subset of $\operatorname{Maps}(E, E U(\ell))$ covering $f$. Equivalently, $E \mathcal{G}=\pi_{*} \mathrm{ev}^{*} E U(\ell)$. Recall that we are using maps in a fixed large Sobolev class; these maps uniformly approximate smooth maps, so that the homotopy types of $E \mathcal{G}$ and $B \mathcal{G}$ are the same for smooth maps or maps in this Sobolev class. Thus $B \mathcal{G}(E)$ and $B \mathcal{G}\left(\pi^{*} E\right)$ are Banach manifolds. For any topological group $G, B G$ admits a partition of unity 
10. Thm. 4.11.2]. Thus $E \mathcal{G}\left(\pi^{*} E\right) \rightarrow B \mathcal{G}\left(\pi^{*} E\right)$ admits a connection with curvature $\Omega \in \Lambda^{2}\left(B \mathcal{G}\left(\pi^{*} E\right), \Gamma \operatorname{End}\left(\pi^{*} E\right)\right)$. Note that the leading order symbol trace on $\Psi \mathrm{DO}_{\leq 0}$ obviously induces a trace $\sigma$ on $\Psi \mathrm{DO}_{\leq 0} / \Psi \mathrm{DO}_{-1} \simeq \Gamma \operatorname{End}\left(\pi^{*} E\right)$. Therefore $E \mathcal{G}\left(\pi^{*} E\right) \rightarrow B \mathcal{G}\left(\pi^{*} E\right)$ has associated de Rham classes $c_{k}^{\text {lo }}\left(E \mathcal{G}\left(\pi^{*} E\right)\right)=\left[\sigma\left(\Omega^{k}\right)\right] \in$ $H^{2 k}\left(B \mathcal{G}\left(\pi^{*} E\right), \mathbb{C}\right)$.

The following definition is natural in light of (4.2).

Definition 4.1. The $\mathrm{k}^{\text {th }}$ leading order Chern class $c_{k}^{\text {lo }}\left(E \Psi D O_{0}^{*}\right)$ is the de Rham cohomology class of $(B m)^{*} c_{k}^{\mathrm{lo}}\left(E \mathcal{G}\left(\pi^{*}(E)\right) \in H^{2 k}\left(B \Psi \mathrm{DO}_{0}^{*}, \mathbb{C}\right)\right.$.

Set $\mathcal{G}=\mathcal{G}(E)$. Let $\mathcal{E} \rightarrow B$ be a $\mathcal{G}$-bundle, classified by a map $g: B \rightarrow B \mathcal{G}$. The maps $j$ and $m \circ j$ in (4.1) are injective, so every $\mathcal{G}(E)$-bundle is both a $\Psi \mathrm{DO}_{0}^{*}(E)$ bundle and a $\mathcal{G}\left(\pi^{*} E\right)$-bundle. We get

$$
c_{k}^{l o}(\mathcal{E})=g^{*} c_{k}^{l o}(E \mathcal{G})=g^{*} B j^{*} c_{k}^{l o}\left(E \Psi D O_{0}^{*}\right)=g^{*} B j^{*} B m^{*} c_{k}^{\text {lo }}\left(E \mathcal{G}\left(\pi^{*}(E)\right) .\right.
$$

This gives an easy criterion to detect cohomology classes for the classifying spaces.

Lemma 4.2. Let $\mathcal{E}$ be a $\mathcal{G}$-bundle with $c_{k}^{\mathrm{lo}}(\mathcal{E}) \neq 0$. Then the cohomology classes $c_{k}^{\mathrm{lo}}(E \mathcal{G}) \in H^{2 k}(B \mathcal{G}, \mathbb{C}), c_{k}^{\mathrm{lo}}\left(E \Psi \mathrm{DO}_{0}^{*}\right) \in H^{2 k}\left(B \Psi \mathrm{DO}_{0}^{*}, \mathbb{C}\right), c_{k}^{\mathrm{lo}}\left(E \mathcal{G}\left(\pi^{*}(E)\right) \in\right.$ $H^{2 k}\left(B \mathcal{G}\left(\pi^{*}(E), \mathbb{C}\right)\right.$ are all nonzero.

As in Remark 2.1, let $\pi: \operatorname{Maps}(N, M) \times N \rightarrow \operatorname{Maps}(N, M)$ be the projection, and for $n \in N$, define $\operatorname{ev}_{n}: \operatorname{Maps}(N, M) \rightarrow M$ by ev $_{n}(f)=\operatorname{ev}(n, f)=f(n)$.

Example 4.3. Let $F \rightarrow M$ be a complex bundle, and set $E=\operatorname{ev}^{*} F \rightarrow \operatorname{Maps}_{f}(N, M) \times$ $M, \mathcal{E}=\pi_{*} \mathrm{ev}^{*} F \rightarrow \operatorname{Maps}_{f}(N, M)$. Here $\operatorname{Maps}_{f}(N, M)$ is the component of a fixed $f: N \rightarrow M$. Then the Lemma applies with $\mathcal{G}=\mathcal{G}\left(f^{*} F\right)$, since $\mathcal{E}_{g}$ is noncanonically isomorphic to $H^{s_{0}} \Gamma\left(f^{*} F\right)$ for all $g \in \operatorname{Maps}_{f}(N, M)$.

Lemma 4.4. EG has a universal connection with connection one-form $\theta^{\text {EG }}$ defined on $s \in \Gamma(E \mathcal{G})$ by

$$
\left(\theta_{Z}^{E \mathcal{G}} s\right)(\gamma)(\alpha)=\left(\left(\mathrm{ev}^{*} \theta^{u}\right)_{(Z, 0)} u_{s}\right)(\gamma, \alpha)
$$

Here $\theta^{u}$ is the universal connection on $\left.E U(k)\right) \rightarrow B U(k)$, and $u_{s}: \operatorname{Maps}(N, M) \times$ $N \rightarrow \mathrm{ev}^{*} E U(k)$ is defined by $u_{s}(f, n)=s(f)(n)$.

Corollary 4.5. The curvature $\Omega^{E \mathcal{G}}$ of $\theta^{E \mathcal{G}}$ satisfies

$$
\Omega^{E \mathcal{G}}(Z, W) s(f)(n)=\mathrm{ev}^{*} \Omega^{u}((Z, 0),(W, 0)) u_{s}(f, n) .
$$

The proofs are in [14, $\S 4]$ for loop spaces $\left(N=S^{1}\right)$ and easily extend. As a result, the leading order Chern classes of gauge bundles are pullbacks of finite dimensional classes.

Lemma 4.6. Fix $n_{0} \in N$. Then

$$
c_{k}^{\mathrm{lo}}(E \mathcal{G})=\operatorname{vol}\left(S^{*} N\right) \cdot \mathrm{ev}_{n_{0}}^{*} c_{k}(E U(\ell)) .
$$

If $\mathcal{F} \rightarrow \operatorname{Maps}(N, M)$ is given by $\mathcal{F}=\pi_{*} \mathrm{ev}^{*} F$ for a bundle $F \rightarrow M$, then

$$
c_{k}^{\mathrm{lo}}(\mathcal{F})=\operatorname{vol}\left(S^{*} N\right) \cdot \mathrm{ev}_{n_{0}}^{*} c_{k}(F) .
$$
of

Proof. For all $n_{0} \in N$, the maps $\mathrm{ev}_{n_{0}}$ are homotopic, so the de Rham class

$$
\int_{S^{*} N} \operatorname{tr} \sigma_{0}\left(\operatorname{ev}_{n_{0}}^{*}\left(\Omega^{u}\right)^{k}\right) d \xi \operatorname{dvol}\left(n_{0}\right)=\operatorname{vol}\left(S^{*} N\right) \cdot \operatorname{tr} \sigma_{0}\left(\operatorname{ev}_{n_{0}}^{*}\left(\Omega^{u}\right)^{k}\right)
$$


is independent of $n_{0}$. Since $\Omega^{u}$ is a multiplication operator, we get

$$
c_{k}^{\mathrm{lo}}(E \mathcal{G})=\operatorname{vol}\left(S^{*} N\right) \cdot\left[\mathrm{ev}_{n_{0}}^{*}\left(\operatorname{tr} \Omega^{u}\right)^{k}\right]=\operatorname{vol}\left(S^{*} N\right) \cdot \mathrm{ev}_{n_{0}}^{*} c_{k}(E U(\ell)) .
$$

The proof for $\mathcal{F}$ is identical.

Note that $\left(\operatorname{vol}\left(S^{*} N\right) \cdot(2 \pi i)^{k}\right) c_{k}^{\mathrm{lo}}(E \mathcal{G}) \in H^{2 k}(B \mathcal{G}, \mathbb{Z})$. It is not clear that we can normalize $c_{k}^{\text {lo }}\left(E \Psi D O_{0}^{*}\right), c_{k}^{\text {lo }}\left(E \mathcal{G}\left(\pi^{*}(E)\right)\right.$ to be integer classes.

We can produce examples of nontrivial $c_{k}^{\mathrm{lo}}\left(\operatorname{Maps}(N, M)=c_{k}^{\mathrm{lo}}(T \operatorname{Maps}(N, M))\right.$ as well as other cohomology classes for $\operatorname{Maps}(N, M)$.

Theorem 4.7. (i) Let $M$ have $c_{k}(M)=c_{k}(T M \otimes \mathbb{C}) \neq 0$. Then

$$
0 \neq c_{k}^{\mathrm{lo}}(\operatorname{Maps}(N, M)) \in H^{2 k}(\operatorname{Maps}(N, M), \mathbb{C}) .
$$

(ii) Let $F^{\ell} \rightarrow M$ be a finite rank Hermitian bundle with $c_{k}(F) \neq 0$. Then

$$
0 \neq c_{k}^{\mathrm{lo}}\left(\pi_{*} \operatorname{ev}^{*} F\right) \in H^{2 k}(\operatorname{Maps}(N, M), \mathbb{C}) .
$$

Proof. (i) Let $h: M \rightarrow B U(m)$ classify $T M \otimes \mathbb{C}$. (Strictly speaking, we take a classifying map into a Grassmannian $B U(m, K)$ of $m$-planes in $\mathbb{C}^{K}$ for $K \gg 0$, so that the target space is a finite dimensional manifold.) For fixed $f \in \operatorname{Maps}(N, M)$, the gauge group $\mathcal{G}$ of $f^{*}(T M \otimes \mathbb{C})$ has

$$
\begin{aligned}
B \mathcal{G} & =\left\{g \in \operatorname{Maps}(N, B U(m)): g^{*} B U(m) \simeq f^{*}(T M \otimes \mathbb{C})\right\} \\
& =\{g \in \operatorname{Maps}(N, B U(m)): g \sim h f\} .
\end{aligned}
$$

Therefore the map

$$
\tilde{h}: \operatorname{Maps}(N, M) \rightarrow \operatorname{Maps}(N, B U(m)), \tilde{h}(f)=h f
$$

classifies $T \operatorname{Maps}(N, M)$, and so for fixed $n \in N$,

$$
c_{k}^{\mathrm{lo}}(\operatorname{Maps}(N, M))=\operatorname{vol}\left(S^{*} N\right) \cdot \tilde{h}^{*} \mathrm{ev}_{n}^{*} c_{k}(E U(m)),
$$

by Lemma 4.6

Let $[a]$ be a $2 k$-cycle with $\left\langle c_{k}(M),[a]\right\rangle \neq 0$. (Here the bracket refers to integration of forms over cycles.) For $i: M \rightarrow \operatorname{Maps}(N, M), i\left(m_{0}\right)(n)=m_{0}$, set $[\tilde{a}]=i_{*}[a]$. Then

$$
\left\langle c_{k}^{\mathrm{lo}}(\operatorname{Maps}(N, M)),[\tilde{a}]\right\rangle=\operatorname{vol}\left(S^{*} N\right) \cdot\left\langle c_{k}(E U(m)), \mathrm{ev}_{n, *} \tilde{h}_{*}[\tilde{a}]\right\rangle .
$$

It is immediate that $\operatorname{ev}_{n} \tilde{h} i=h$, so $\operatorname{ev}_{n, *} \tilde{h}_{*}[\tilde{a}]=h_{*}[a]$. Therefore

$$
\begin{aligned}
\left\langle c_{k}^{\mathrm{lo}}(\operatorname{Maps}(N, M)),[\tilde{a}]\right\rangle & =\operatorname{vol}\left(S^{*} N\right) \cdot\left\langle h^{*} c_{k}(E U(m)),[a]\right\rangle \\
& =\operatorname{vol}\left(S^{*} N\right) \cdot\left\langle c_{k}(T M \otimes \mathbb{C}),[a]\right\rangle \\
& \neq 0 .
\end{aligned}
$$

(ii) Let $h: M \rightarrow B U(\ell)$ classify $F$. As above, $\tilde{h}$ classifies $\mathcal{F}=\pi_{*} \mathrm{ev}^{*} F$. Thus

$$
c_{k}^{\mathrm{lo}}(\mathcal{F})=\tilde{h}^{*} c_{k}^{\mathrm{lo}}(E \mathcal{G})=\operatorname{vol}\left(S^{*} N\right) \cdot \tilde{h}^{*} \operatorname{ev}_{n}^{*} c_{k}(E U(\ell))
$$

by Lemma 4.6. As in (i), we get

$$
\left\langle c_{k}^{\mathrm{lo}}(\mathcal{F}),[\tilde{a}]\right\rangle=\left\langle c_{k}(F),[a]\right\rangle \neq 0
$$

for some cycle $[a]$. Alternatively, we can use the last statement in Lemma 3.13 and $\mathrm{ev}_{n} i=\mathrm{Id}$ to reach the same conclusion.

In this proof, the cycle $[\tilde{a}]$ has image in $\operatorname{Maps}_{c}(N, M)$, the component of the constant maps, so the result is really about bundles over this component. We can improve this to cover all components. 
Corollary 4.8. For $f \in \operatorname{Maps}(N, M)$, let $\operatorname{Maps}_{f}(N, M)$ denote the connected component of $f$. Let $F^{\ell} \rightarrow M$ be a finite rank Hermitian bundle with $c_{k}(F) \neq 0$. Assume that $M$ is connected. Then

$$
0 \neq c_{k}^{\mathrm{lo}}\left(\pi_{*} \mathrm{ev}^{*} F\right) \in H^{2 k}\left(\operatorname{Maps}_{f}(N, M), \mathbb{C}\right) .
$$

Proof. We claim that for fixed $n_{0} \in N$ the map

$$
\operatorname{ev}_{n_{0}, *}: H_{*}\left(\operatorname{Maps}_{f}(N, M), \mathbb{C}\right) \rightarrow H_{*}(M, \mathbb{C})
$$

is surjective.

As a first step, we show that for a fixed $m_{0} \in M$, we can homotop $f$ to a map $\tilde{f}$ with $\tilde{f}\left(n_{0}\right)=m_{0}$. By the tubular neighborhood theorem applied to the one-manifold/path from $f\left(n_{0}\right)$ to $m_{0}$, there exists a coordinate chart $W=\phi\left(\mathbb{R}^{n}\right)$ containing $f\left(n_{0}\right)$ and $m_{0}$. Take small coordinate balls $U$ containing $\phi^{-1}\left(f\left(n_{0}\right)\right)$ and $V$ containing $\phi^{-1}\left(m_{0}\right)$, such that $V$ is a translate $\vec{T}+U$ of with $\vec{T}=\phi^{-1}\left(m_{0}\right)-$ $\phi^{-1}\left(n_{0}\right)$. We may assume that $U$ is a ball of radius $r$ centered at $\phi^{-1}\left(n_{0}\right)$. Let $\psi:[0, r] \rightarrow \mathbb{R}$ be a nonnegative bump functions which is one near zero and zero near $r$. Define $f_{t}: N \rightarrow M$ by

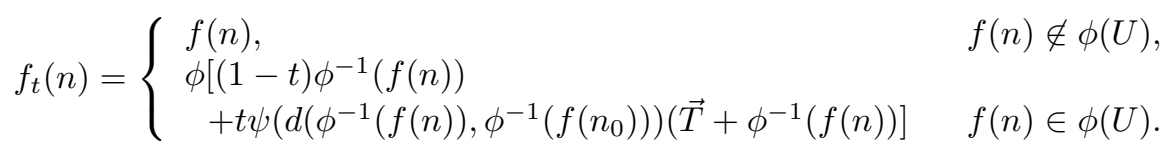

In other words, $f_{t}=f$ outside $f^{-1}(\phi(U))$ and moves points $f(n) \in \phi(U)$ towards $\phi(V)$, with $f_{t}\left(n_{0}\right)$ moving $f\left(n_{0}\right)$ to $m_{0}$. Now set $\tilde{f}=f_{1}$.

Take a $k$-cycle $\sum r_{i} \sigma_{i}$ in $M$. Let $\Delta^{k}=\left\{\left(x_{1}, \ldots, x_{k}\right): x_{i} \geq 0, \sum x_{i} \leq 1\right\}$ be the standard $k$-simplex. By subdivision, we may assume that each $\sigma_{i}\left(\Delta^{k}\right)$ is in a coordinate patch $V^{\prime}=V_{i}^{\prime}=\phi\left(V_{i}\right)$ in the notation above. Construct the corresponding neighborhood $U^{\prime}=\phi\left(U_{i}\right)$ of $f\left(n_{0}\right)$. Set $m_{0}=\sigma_{i}(\overrightarrow{0})$. Take a map $\alpha_{i}: \Delta^{k} \rightarrow \operatorname{Maps}_{f}(N, M)$ with $\alpha(\overrightarrow{0})=f$ and $\alpha_{i}(x)\left(n_{0}\right) \in U^{\prime}$ for all $x \in \Delta^{k}$. By suitably modifying the bump function to vanish near $d\left(\phi^{-1}\left(\alpha_{i}(x)\left(n_{0}\right)\right), \partial U\right)$, we can form a simplex $\tilde{\alpha}_{i}: \Delta^{k} \rightarrow \operatorname{Maps}_{f}(N, M)$ with $\tilde{\alpha}_{i}(x)\left(n_{0}\right)=\sigma_{i}(x)$ and $\tilde{\alpha}_{i}(\overrightarrow{0})=\tilde{f}$.

Clearly $\sum r_{i} \tilde{\alpha}_{i}$ is a cycle in $\operatorname{Maps}_{f}(N, M)$ with $\operatorname{ev}_{n_{0}, *}\left[\sum r_{i} \tilde{\alpha}_{i}\right]=\left[\sum_{i} r_{i} \sigma_{i}\right]$. This finishes the claim. Note that this construction is an ad hoc replacement for the map $i$ in the last theorem.

Pick a cycle $[b] \in H_{2 k}(M, \mathbb{C})$ such that $\left\langle c_{k}(F),[b]\right\rangle \neq 0$. Pick $[\tilde{b}] \in$ $H_{2 k}\left(\operatorname{Maps}_{f}(N, M), \mathbb{C}\right.$ with $\operatorname{ev}_{n_{0}, *}[\tilde{b}]=[b]$. Then

$$
\left\langle c_{k}^{\mathrm{lo}}\left(\pi_{*} \mathrm{ev}^{*} F\right),[\tilde{b}]\right\rangle=\operatorname{vol}\left(S^{*} N\right) \cdot\left\langle c_{k}(F), \mathrm{ev}_{n_{0}, *}[b]\right\rangle \neq 0
$$

as in Theorem 4.7 (ii).

This gives information about the cohomology rings of the various classifying spaces. Recall that we are working with either the Fréchet or the norm topology on $\Psi D O_{0}^{*}$.

Proposition 4.9. Fix a closed manifold $N$ and a connected manifold $M$. Let $F^{\ell} \rightarrow M$ be a finite rank Hermitian bundle, choose $f: N \rightarrow M$, and let $\mathcal{G}, \Psi \mathrm{DO}_{0}^{*}$, refer to the gauge groups and $\Psi \mathrm{DO}$ groups acting on sections of $F$. Let $H_{F}^{*}(M, \mathbb{C})$ be the subring of $H^{*}(M, \mathbb{C})$ generated by the Chern classes of $F$. Then for $X=$ $B \mathcal{G}, B \Psi \mathrm{DO}_{0}^{*}, B \mathcal{G}\left(\pi^{*} F\right)$, there is a surjective map from $H^{*}(X, \mathbb{C})$ to an isomorphic copy of $H_{F}^{*}(M, \mathbb{C})$ in $H^{*}\left(\operatorname{Maps}_{f}(N, M), \mathbb{C}\right)$, where $\operatorname{Maps}_{f}(N, M)$ is the component of $f$ in $\operatorname{Maps}(N, M)$. 
Proof. Set $\mathcal{F}=\pi_{*} e v^{*} F$. The proof of Corollary 4.8 shows that if a polynomial $p\left(c_{0}^{\mathrm{lo}}(\mathcal{F}), \ldots, c_{\ell}^{\mathrm{lo}}(\mathcal{F})\right) \in H^{*}\left(\operatorname{Maps}_{f}(N, M), \mathbb{C}\right)$ vanishes, then $p\left(c_{0}(F), \ldots, c_{\ell}(F)\right)$

$=0$. Thus $H_{F}^{*}(M, \mathbb{C})$ injects into $H^{*}\left(\operatorname{Maps}_{f}(N, M), \mathbb{C}\right)$, for any $N$, with image the ring generated by $c_{k}^{\text {lo }}(\mathcal{F}), k \leq \ell$. Let $h$ classify $F$. In the notation of (4.2) and the previous theorem, we have

$$
c_{k}^{\mathrm{lo}}(\mathcal{F})=(B j \circ B m \circ \tilde{h})^{*} c_{k}^{\mathrm{lo}}\left(E \mathcal{G}\left(\pi^{*} F\right)\right)=(B m \circ \tilde{h})^{*} c_{k}^{\mathrm{lo}}\left(E \Psi \mathrm{DO}_{0}^{*}\right)=\tilde{h}^{*} c_{k}^{\mathrm{lo}}(E \mathcal{G}) .
$$

The surjectivity of $H^{*}(X, \mathbb{C}) \rightarrow \operatorname{Im}\left(H_{F}^{*}(M, \mathbb{C})\right)$ is now immediate.

This gives the result on the cohomology of $B \mathcal{G}, B \Psi \mathrm{DO}_{0}^{*}, B \mathcal{G}\left(\pi^{*}(E)\right.$ stated in the Introduction.

Theorem 4.10. Let $E^{\ell} \rightarrow N$ be a finite rank Hermitian bundle, and let $\mathcal{G}, \Psi \mathrm{DO}_{0}^{*}$, refer to the gauge groups and $\Psi \mathrm{DO}$ groups acting on sections of $E$. Then for $X=$ $B \mathcal{G}, B \Psi \mathrm{DO}_{0}^{*}, B \mathcal{G}\left(\pi^{*} E\right)$, there is a surjective map from $H^{*}(X, \mathbb{C})$ to the polynomial algebra $H^{*}(B U(\ell), \mathbb{C})=\mathbb{C}\left[c_{1}(E U(\ell)), \ldots, c_{\ell}(E U(\ell))\right]$.

Proof. Let $M=B U(\ell, K)$ be the Grassmannian of $\ell$-planes in $\mathbb{C}^{K}$, for $K \gg 0$, let $F=E U(\ell, K)$, and let $f: N \rightarrow M$ classify $E$. On the component $\operatorname{Maps}_{f}(N, M)$ of $f, \mathcal{E}=\pi_{*} \mathrm{ev}^{*} E U(\ell, K)$ has structure group $\mathcal{G}\left(f^{*} E U(\ell, K)\right)=$ $\mathcal{G}(E) . H^{*}(M, \mathbb{C})$ is a polynomial algebra with generators $c_{1}(E U(\ell, K)), \ldots$, $c_{\ell}(E U(\ell, K))$ truncated above $\operatorname{dim}(M)=\ell(K-\ell)$. By the previous proposition, $H^{*}(X, \mathbb{C})$ surjects onto this algebra. Letting $K$ go to infinity finishes the proof.

Remark 4.11. (i) A proof of Theorem 4.10 for $H^{*}(B \mathcal{G}, \mathbb{C})$ that avoids most of the analysis can be extracted from Lemma 4.6 through Proposition 4.9

(ii) $E \mathcal{G}(E) \rightarrow B \mathcal{G}(E)$ is trivial as a $G L(\mathcal{H})$-bundle by Kuiper's theorem. However, $E \mathcal{G}(E) \rightarrow B \mathcal{G}(E)$ is nontrivial as a $\mathcal{G}(E)$-bundle, as it has nontrivial leading order characteristic classes.

We conclude with a result that complements Rochon's calculations of the homotopy groups of $\Psi \mathrm{DO}_{0}^{*}[\mathbf{1 6}$.

Corollary 4.12. In the setup of the Proposition 4.9 , if $H_{F}^{*}(M, \mathbb{C})$ is nontrivial, then $\operatorname{Ell}^{*}(F)$ is not a deformation retract of $\Psi \mathrm{DO}_{0}^{*}(F)$.

Proof. Assume Ell ${ }^{*}$ is a deformation retract of $\Psi \mathrm{DO}_{0}^{*}$. Then every $\Psi \mathrm{DO}_{0^{-}}^{*}$ bundle admits a reduction to a Ell ${ }^{*}$-bundle. Let $\mathcal{E} \rightarrow B$ be a Ell*-bundle admitting a connection. Lie $\left(\mathrm{Ell}^{*}\right)$ is the algebra of negative order $\Psi$ DOs, so the connection and curvature forms have vanishing leading order Chern classes. For $B=\operatorname{Maps}(M, M)$ and $f=\mathrm{id}$, the proof of Proposition 4.9 gives an injection of $H_{F}(M, \mathbb{C})$ into the subring of $H^{*}(\operatorname{Maps}(M, M), \mathbb{C})$ generated by the leading order Chern classes. This is a contradiction.

\section{References}

1. Atiyah, M. and Bott, R., Yang-Mills equations on Riemann surfaces, Phil. Trans. Roy. Soc. London Series A 308 (1982), 524-615.

2. E. Beggs, The de Rham complex on infinite dimensional manifolds, Quarterly J. of Math. 38 (1987), 131-154.

3. J. M. Bismut, The Atiyah-Singer index theorem for families of Dirac operators: two heat equation proofs, Inventiones Math. 83 (1986), 91-151.

4. Booss-Bavnek, B. and Wojciechowski, K., Elliptic Boundary Problems for Dirac Operators, Birkhäuser, Boston, 1993. 
5. Donaldson, S. K. and Kronheimer, P. B., The Geometry of Four-Manifolds, Oxford U. Press, Oxford, 1990.

6. J. Eells, A setting for global analysis, Bull. Amer. Math. Soc 72 (1966), 751-807.

7. Gilkey, P. B., Invariance Theory, the Heat Equation, and the Atiyah-Singer Index Theorem, Publish or Perish, Wilmington, DE, 1984.

8. R. Hamilton, Nash-Moser implicit function theorems, Bull. Amer. Math. Soc. 7 (1986), 65222 .

9. L. Hörmander, The Analysis of Linear Partial Differential Operators III: Pseudo-Differential Operators, Springer-Verlag, New York, 2007.

10. D. Husemoller, Fibre bundles, Springer-Verlag, New York, 1966.

11. J.-M. Lescure and S. Paycha, Uniqueness of multiplicative determinants on elliptic pseudodifferential operators, Proc. London Math. Soc. 94 (2007), 772-812.

12. Maeda, Y., Rosenberg, S., and Torres-Ardila, F., Riemannian geometry on loop spaces, arXiv:0705.1008.

13. Omori, H., Infinite-Dimensional Lie Groups, A.M.S., Providence, RI, 1997.

14. Paycha, S. and Rosenberg, S., Chern-Weil constructions on $\Psi$ DO bundles, math.DG/0301185.

15. , Traces and characteristic classes on loop spaces, Infinite Dimensional Groups and Manifolds (T. Wurzbacher, ed.), Walter de Gruyter, 2004, pp. 185-212.

16. F. Rochon, Sur la topologie de l'espace des opérateurs pseudodifférentiels inversibles d'ordre 0, Ann. Inst. Fourier 58 (2008), 29-62.

17. Shubin, M., Pseudodifferential Operators and Spectral Theory, Springer-Verlag, New York, 2001.

18. Spanier, E., Algebraic Topology, McGraw-Hill, New York, 1966.

Department of Mathematics and Statistics, Boston University

E-mail address: alh@bu.edu

Department of Mathematics and Statistics, Boston University

E-mail address: sr@math.bu.edu

Department of Mathematics, King's College

E-mail address: simon.scott@kcl.ac.uk

Metropolitan College, Boston University

E-mail address: fatorres@math.bu.edu 